\title{
Construction of a cDNA library for miniature pig mandibular deciduous molars
}

Tieli Song ${ }^{1,2 \dagger}$, Tingting $\mathrm{Wu}^{1+}$, Fulan Wei ${ }^{1}$, Ang Li ${ }^{1}$, Fu Wang ${ }^{1}$, Yilin Xie ${ }^{1}$, Dayong Liu', Zhipeng Fan ${ }^{1}$, Xuejiu Wang ${ }^{1}$, Shan Cheng ${ }^{3}$, Chunmei Zhang ${ }^{1}$, Junqi $\mathrm{He}^{3}$ and Songlin Wang ${ }^{1,3^{*}}$

\begin{abstract}
Background: The miniature pig provides an excellent experimental model for tooth morphogenesis because its diphyodont and heterodont dentition resembles that of humans. However, little information is available on the process of tooth development or the exact molecular mechanisms controlling tooth development in miniature pigs or humans. Thus, the analysis of gene expression related to each stage of tooth development is very important.

Results: In our study, after serial sections were made, the development of the crown of the miniature pigs' mandibular deciduous molar could be divided into five main phases: dental lamina stage (E33-E35), bud stage (E35-E40), cap stage (E40-E50), early bell stage (E50-E60), and late bell stage (E60-E65). Total RNA was isolated from the tooth germ of miniature pig embryos at E35, E45, E50, and E60, and a cDNA library was constructed. Then, we identified CDNA sequences on a large scale screen for CDNA profiles in the developing mandibular deciduous molars (E35, E45, E50, and E60) of miniature pigs using Illumina Solexa deep sequencing. Microarray assay was used to detect the expression of genes. Lastly, through Unigene sequence analysis and cDNA expression pattern analysis at E45 and E60, we found that 12 up-regulated and 15 down-regulated genes during the four periods are highly conserved genes homologous with known Homo sapiens genes. Furthermore, there were 6 down-regulated and 2 up-regulated genes in the miniature pig that were highly homologous to Homo sapiens genes compared with those in the mouse.
\end{abstract}

Conclusion: Our results not only identify the specific transcriptome and cDNA profile in developing mandibular deciduous molars of the miniature pig, but also provide useful information for investigating the molecular mechanism of tooth development in the miniature pig.

Keywords: Tooth, Development, Histology, Unigene, Sequence, Miniature pig

\section{Background}

The pig is a large animal species suitable not only for meat production, but also as a model organism for comparative genomics and biomedical studies [1-6]. Due to the similarity of the dental and jaw bone system between human and pigs [7-9], using swine in dental biomedical research has increased in recent years, including research into dental implants, irradiation damage to parotid glands, bio-root

\footnotetext{
*Correspondence: slwang@ccmu.edu.cn

${ }^{\dagger}$ Equal contributors

${ }^{1}$ Molecular Laboratory for Gene Therapy and Tooth Regeneration, Beijing Key Laboratory of Tooth Regeneration and Function Reconstruction, Capital Medical University School of Stomatology, Tian Tan Xi Li No.4, Beijing 100050, China

${ }^{3}$ Department of Biochemistry and Molecular Biology, Capital Medical University School of Basic Medical Sciences, Beijing 100069, China Full list of author information is available at the end of the article
}

regeneration, osteoradionecrosis, and bisphosphonaterelated osteonecrosis, etc. [10-16].

The mouse is the most widely used animal model for studying tooth development. Almost all known molecular mechanisms of tooth formation and mineralization are derived indirectly or directly from studies of murine models [17-19]. However, mouse teeth are different from those of humans in both number and morphology, with only one dentition present throughout the mouse life cycle and a complete absence of canines and premolars [20]. Miniature pigs have both deciduous and permanent dentition, and all tooth types found in humans are present in pigs. However, detailed descriptive information concerning tooth development in the pig is lacking. Recently, our group has been dedicated to investigating the complicated mechanism of tooth development in

\section{Biomed Central}


miniature pigs, including the mRNA expression profiles of developing deciduous molar tooth [21], and the timing and sequencing of tooth replacement [22]. Other groups also reported that early morphogenesis of heterodont dentition can be divided into four significant stages in miniature pigs [23]. The purpose of the present study was to identify and classify the early stages of odontogenesis in miniature pig's deciduous molar teeth, focusing on the differential expression of cDNAs during typical periods of tooth development. We also compared the genes from the E45 to E60 time course during tooth development with those of known Homo sapiens genes, and aimed to obtain basic information about their development for further molecular studies. We found that 12 up-regulated and 15 down-regulated genes may be involved in the miniature pig's tooth development. We also found there were 6 down-regulated and 2 up-regulated genes with high homology to those in Homo sapiens, and compared these with those in mouse.

\section{Methods}

\section{Ethics statement}

Pregnant Wuzhishan miniature pigs were obtained from the Institute of Animal Science of the Chinese Agriculture University. Experiments were performed according to the Regulations for the Administration of Affairs Concerning Experimental Animals (Ministry of Science and Technology, China, revised in June 2004), and approved by the Animal Care and Use Committees of Capital Medical University, Beijing, China under permit No. CMU-B20100106. Animals were allowed access to food and water ad libitum under normal conditions and humanely sacrificed as necessary to ameliorate suffering. In brief, pregnant sows were anesthetized with a combination of $6 \mathrm{mg} / \mathrm{kg}$ ketamine chloride and $0.6 \mathrm{mg} / \mathrm{kg}$ xylazine, and pregnancy and the fetal state roughly determined by B-mode ultrasonography. After removing the fetuses by cesarean section, the pregnant sows were sacrificed by over-anesthetization.

\section{Preparation of tissues and histological staining}

Developing miniature pig embryos were obtained by hysterectomy at embryonic days 30 (E30), E35, E40, E45, E50, E55, E60, and E65 according to the developmental progression of deciduous dentition in pigs [24]. After surgically removing the fetuses, germ tissue samples from deciduous molar teeth were removed from the mandibles under a microscope. The first mandibular molar could be obtained from E30. The second mandibular molar could be obtained from E35. The third mandibular molar could be obtained from E45. So the first deciduous mandibular molars were used in all studies. The samples were immediately frozen in liquid nitrogen and stored separately at $-80^{\circ} \mathrm{C}$ until used for analysis. At least five miniature pig embryos were used for each evaluation. Specimens for the histological study were chosen by random selection from each specific age group litter. Embryo mandibles were separated and preserved in $4 \%$ paraformaldehyde. Mandible specimens from E30 were placed in EDTA bone decalcifying agent. Serial sections were made of the mandibular deciduous molar region. The tissues were mounted and stained with hematoxylin and eosin.

\section{RNA sample preparation and CDNA library establishment} Mandibular deciduous molar germs from E35, E45, E50, and E60 miniature pig embryos were excised and total RNA was extracted with an RNA purification kit (QIAGEN, Germany). RNA was then mixed in equal amounts from four different developmental time points. Oligo dT cellulose (MicroFast Track, Invitrogen, CA) was used as a template to synthesize first-strand cDNA. The cDNA library was constructed using the SMART cDNA Library Construction Kit (Clontech, CA). The obtained double-stranded (ds)-cDNA was purified using the QIAquick PCR Purification Kit (QIAGEN, Germany), then normalized with the DSN (duplex-specific nuclease) using the Trimmer-Direct Kit (Evrogen, Moscow, Russia). The normalized cDNAs were digested with Sfi I restriction enzyme, size fractionated (1-3 kb), directionally ligated into pDNR-LIB, and transformed into E. coli $\mathrm{DH} 10 \mathrm{~B}$ by electroporation. The cDNA library was plated on LB plates with X-gal, isopropyl-D-thiogalactopyranoside, and ampicillin. Thirty white colonies were randomly selected for identification of cDNA inserts in the recombinants to estimate the recombination efficiency. Exact same samples were used for both microarray and qRT-PCR.

\section{Microarray procedures}

Microarray targets were prepared from each stage. RNA labelling, hybridization and scanning were conducted by a commercial Affymetrix array service (Institut de Recerca Hospital Universitari Vall d'Hebron, Barcelona, Spain). Reverse transcription of RNA and synthesis of biotinlabelled cRNA with one round of amplification were carried out following the standard Affymetrix onecycle protocol according to the manufacturer's instructions. Samples were hybridized to the Affymetrix $24 \mathrm{~K}$ Genechip ${ }^{\circ}$ Porcine Genome Array (Affymetrix, Santa Clara, CA, USA). Data analysis was performed with Bioconductor implemented in R 2.6.0 (http://cran.r-project.org/).

\section{Quantitative real-time RT-PCR}

Total RNA reversely transcribed into cDNA using the PrimeScriptTM PT Reagent Kit (TaKaRa, Dalian, China). Amplifications of target genes were performed by realtime quantitative PCR (qPCR) using the cDNA as template, the specific primers and the $\mathrm{SYBR}^{\circ}$ PrimeScript $^{\circ}$ RT-PCR Kit (Takara) on an ABI PRISM 7900 Real Time 
PCR System (Applied Biosystems, Carlsbad, USA). PCR amplifications were performed in duplicate at $95^{\circ} \mathrm{C}$ for $15 \mathrm{sec}$, and subjected to 40 cycles of $95^{\circ} \mathrm{C}$ for $5 \mathrm{sec} 60^{\circ} \mathrm{C}$ for $30 \mathrm{sec}$, and $95^{\circ} \mathrm{C}$ for $15 \mathrm{sec} 60^{\circ} \mathrm{C}$ for $15 \mathrm{sec} 95^{\circ} \mathrm{C}$ for $15 \mathrm{sec}$. The primers used are shown in Additional file 1. The relative levels of target genes expression to the control of E45 were quantified. The relative levels of target gene mRNA transcripts to control $\beta$-actin were determined by $2^{-\Delta \Delta C t}$.

CDNA library sequencing, data processing, sequence analysis After cDNA library identification, large-scale plasmid extraction and sequencing were performed for generation of expressed sequence tags (ESTs). High-quality
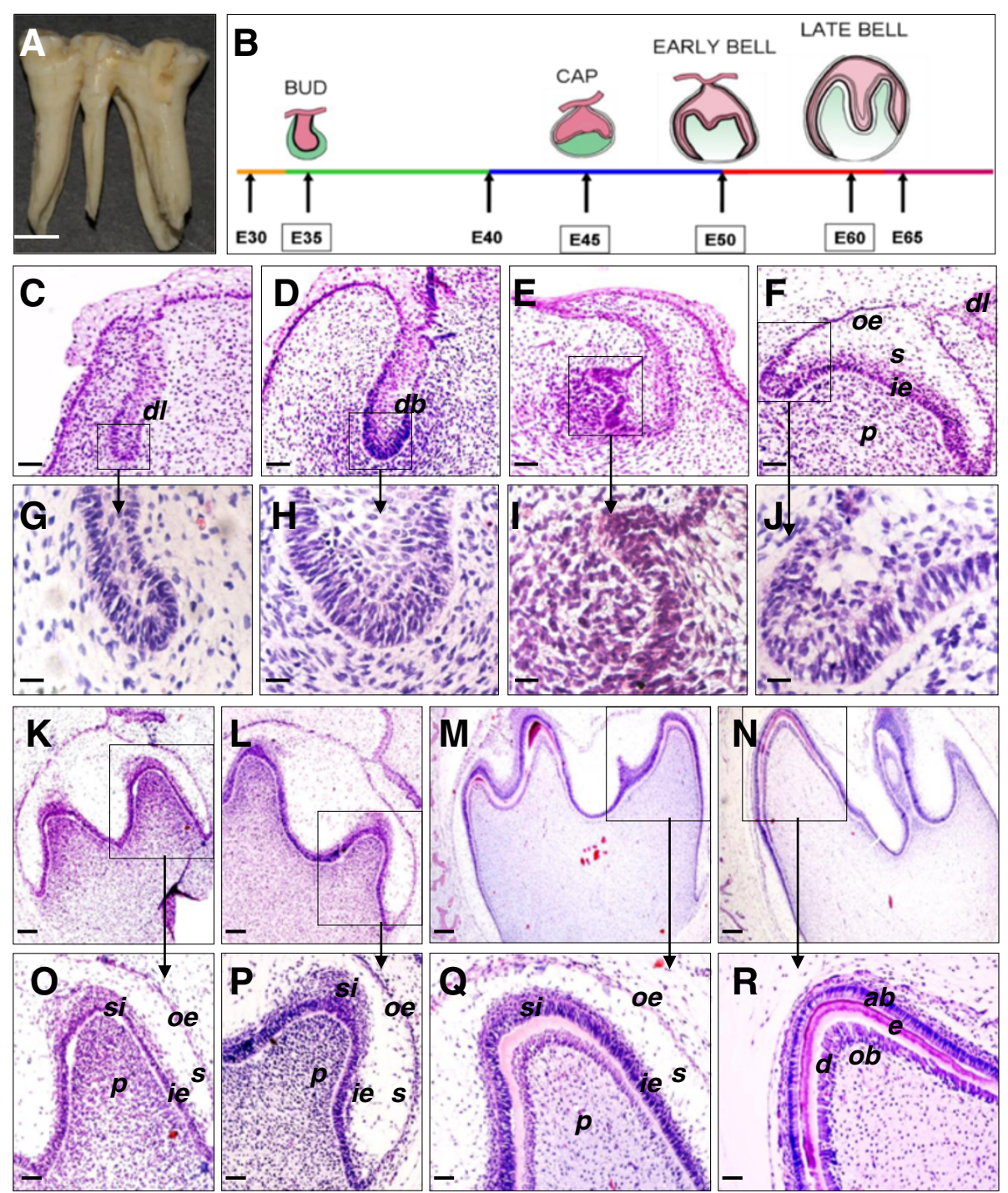

Figure 1 Histology and stage of the developing miniature pig mandibular deciduous molar. (A) Normal mandibular deciduous molar with 5 or 6 main cusps and 5 roots. (B) Developmental stages of the mandibular deciduous molar in the miniature pig. The relatively typical points in time are E35 (bud stage), E45 (cap stage), E50 (early bell stage), and E60 (late bell stage). (C, G) The epithelium grew into the mesenchyme to form the dental lamina $(d)$ at E30. (D, H) The dental lamina formed the dental bud $(d b)$ at E35. (E, I) The peripheral cells of the enamel organ extended outside at E40. $(\mathbf{F}, \mathbf{J})$ A typical cap stage appeared at E45, with differentiation of the outer enamel epithelial cells (oe), inner enamel epithelial cells (ie), and stellate reticular cells $(s)$. The dental papilla $(p)$ could also be observed. $(\mathbf{K}, \mathbf{0})$ At E50, the typical early bell stage was observed. The cusp morphology could be seen at the junction of the inner enamel epithelium and dental papilla. The stratum intermedium (si) appeared between the inner enamel epithelium and the stellate reticulum. (L, P) Morphological findings at E55. (M, Q) By E60, the molar reached late bell stage. In the cusp region, dental epithelial cells and mesenchymal cells were polarized and cells lengthened to become pre-ameloblast and pre-odontoblast. (N, R) Continuous and intact ameloblasts (ab), enamel (e), dentin (d), and odontoblasts (ob) were observed in the molar cusp at E65. Scale bars: $5 \mathrm{~mm}$ in A, $50 \mu \mathrm{m}$ in C-F and O-R, $20 \mu \mathrm{m}$ in G-J, and $100 \mu \mathrm{m}$ in K-N. 
ESTs were assembled into unigenes by phrap_0.990329 software. The unigene sequences were performed with E-values of less than $10^{-5}$ on the GenBank database according to the BLAST Search program (ftp://ftp.ncbi. nih.gov/blast/db/FASTA/). The unigenes were compared with annotations through the Gene Ontology Consortium using Interpro2GO. All ESTs were sequenced and analyzed at a commercial facility (BGI LifeTech Co. Ltd, Beijing, China). If the unigene sequence was more than $100 \mathrm{bp}$ and its homology greater than $90 \%$ with a known functional pig gene, this gene was annotated in the pig genes. Then, if the sequence had high homology to a known gene in other species (E-values $<10^{-5}$ ), it was assumed that the gene is an orthologue of the comparator gene.

\section{Statistical analysis}

Data of qRT-PCR are expressed as mean \pm SEM. Data were analyzed by one-way analysis of variance. Multiple comparison between the groups was performed by using Bonferroni post-tests method. A p value of less than 0.05 was considered statistically significant. Statistical analysis

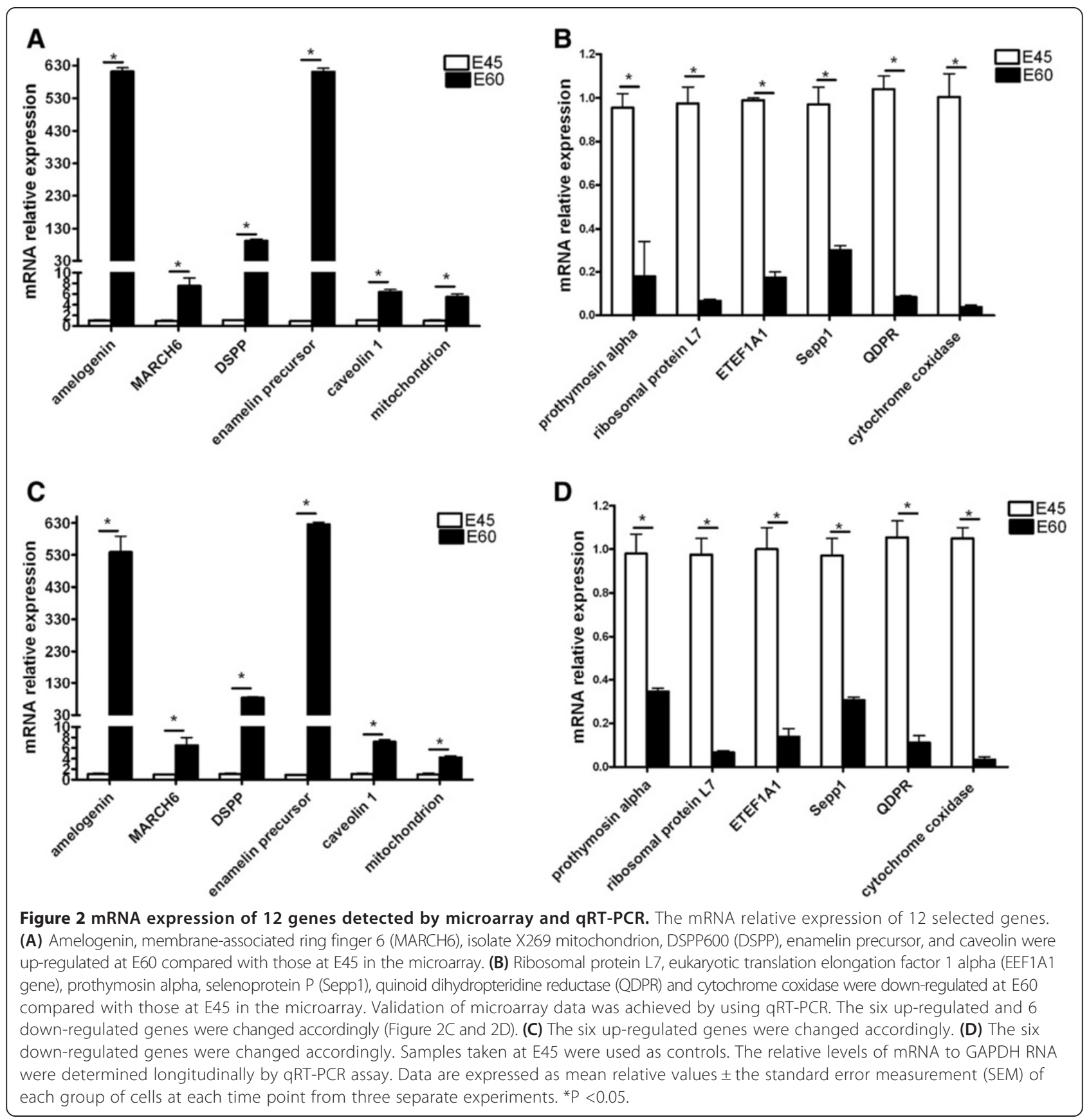


Table 1 A summary of ESTs and unigene analysis

\begin{tabular}{lcc}
\hline Description & Number & Percentage \\
\hline Total number of EST sequences & 20,065 & \\
Number of high quality sequences & 17,520 & $87.3^{1}$ \\
Number of singletons & 11,709 & $58.3^{1}$ \\
Number of contigs & 2,198 & $10.9^{1}$ \\
Number of unigenes & 13,907 & $69.3^{1}$ \\
Number of unigenes with BLAST hits & 10,883 & $78.3^{2}$ \\
Number of unknown unigenes & 3,024 & $21.7^{2}$ \\
\hline
\end{tabular}

Unigene Size: The Number of ESTs in Unigene, ${ }^{1}$ percentage of all ESTs,

${ }^{2}$ percentage of unigenes.

was carried out using StatView 5.0 software (SAS Institute, Cary, NC) and GraphPad Prism 4.0 software.

\section{Results}

Development stages and histological characterization of miniature pig mandibular deciduous molars

Mandibular deciduous molar germs from E30 to E65 miniature pig embryos were excised (Additional file 2). Normal mandibular deciduous molars of miniature pigs have five or six main cusps and five roots (Figure 1A). Figure 1B shows the developmental stages of the mandibular deciduous molars. Development progressed as follows:

E30: In the E30 embryonic mandible, the oral epithelium thickened and extended to form the dental lamina (Figure 1C, G). E35: E35 samples showed hyperplasia of the lamina epithelium cells to form the primary enamel organ, meaning that the typical bud stage was observed (Figure 1D, H). The mesenchymal cells surrounding the bud clearly gathered. The placode was identified between the epithelium and the mesenchyme. E40: In the E40 mandibular region, the molar remained in the bud stage, but minor changes were seen at this time (Figure 1E, I). The peripheral cells of the enamel organ had now extended outside of the bud. E45: The typical cap stage for this molar did not appear until E45. At this time, the entire enamel looked like a cap (Figure 1F, J). More notable cell differentiation was present than at E40. Four cell types were identified; outer enamel epithelium, inner enamel epithelium, stellate reticulum, and dental papilla. The dental sac could also be observed. E50: E50 embryos showed the typical appearance of the early bell stage of this molar (Figure $1 \mathrm{~K}, \mathrm{O}$ ). The dental papilla was larger than during the cap stage, whereas there were no morphological changes of dental papilla cells. The cusp morphology could be seen at the junction of the inner enamel epithelium and dental papilla. Inner enamel epithelial cells near the cusp region became stylolitic in shape, with the nucleolus far from the basalis. The stellate reticulum had sufficiently developed and the stratum intermedium appeared between the inner enamel epithelium and the stellate reticulum. E55: At this stage, there were no further changes except the adoption of a highly stylolitic shape by the inner enamel epithelial cells near the cusp region (Figure 1L, P). E60: By E60, the deciduous molar had reached the late bell stage of development (Figure 1M, Q). In the cusp region, dental epithelial cells and mesenchymal cells were polarized, and the cells lengthened to become preameloblast and pre-odontoblast. At the same time, the pink matrix was seen in the cusp region. E65: At E65, continuous and intact ameloblasts, enamel, dentin, and odontoblasts were observed in the molar cusp (Figure 1N, R).

Taken together, the crown development of miniature pigs' mandibular deciduous molar were divided into five main periods as follows (Figure 1B): the dental lamina stage (E33-E35), bud stage (E35-E40), cap stage (E40-E50), early bell stage (E50-E60), and late bell stage (E60-E65). The relatively typical time points are E35 (bud), E45 (cap), E50 (early bell), and E60 (late bell).

\section{Verification of gene expression}

Amelogenin, membrane-associated ring finger 6 (MARCH6), isolate X269 mitochondrion, DSPP600 (DSPP), enamelin precursor, and caveolin were up-
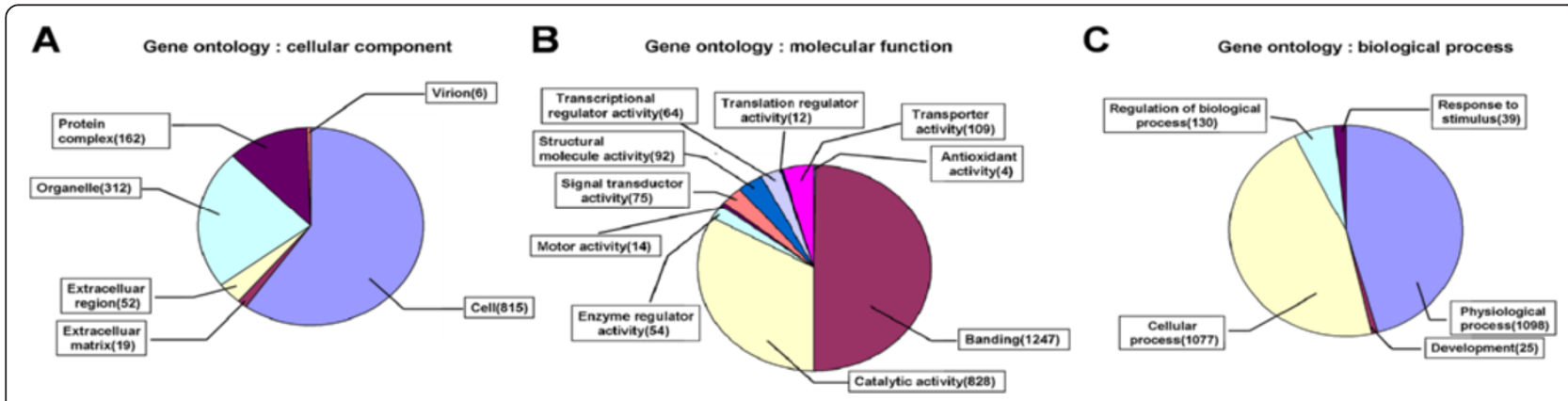

Figure 3 Gene ontology (GO) graphs. The unigene sequences were annotated using Interpro2GO software and included in the graphs. Each of the three categories is presented, including the cellular component (A), molecular function (B), and biological processes (C). 
regulated at E45 compared with those at E60 in the microarray (Figure 2A). Ribosomal protein L7, eukaryotic translation elongation factor 1 alpha (EEF1A1 gene), prothymosin alpha, selenoprotein P (Sepp1), quinoid dihydropteridine reductase (QDPR) and cytochrome coxidase were down-regulated at E45 compared with those at E60 in the microarray (Figure 2B). Validation of microarray data was achieved by using qRTPCR. The six up-regulated and down-regulated genes were changed accordingly (Figure $2 \mathrm{C}$ and $2 \mathrm{D}$ ). The mRNA fold changes of all representative mRNAs were consistent with those in the normalized microarray data.

\section{cDNA library overview}

Crown development in the miniature pig's mandibular deciduous molars could be divided into four relatively typical periods as noted above. The mandibular deciduous molar germ cells were excised from miniature pig embryos at the E35, E45, E50, and E60 time points.

Total RNA from each period was used as template to synthesize cDNA and construct a cDNA library
(Additional file 3 and Additional file 4). The titer of the unamplified cDNA library was approximately $3.0 \times$ $10^{5} \mathrm{cfu} / \mathrm{mL}$. A library comparison showed that all of the 30 selected clones had insert fragments, suggesting that the recombination rate was nearly 100\% (Additional file 5). The primary cDNA library was used to generate ESTs. Twenty-three thousand and six hundred independent white clones were picked randomly for EST sequencing. A total of 20,065 ESTs were sequenced from the cDNA library. After removing the vector sequences and low-quality sequences (EST length less than $100 \mathrm{bp}), 17,520$ high-quality sequences were obtained with an average length of $441.61 \mathrm{bp}$, ranging from 100 to 681 nucleotides in length. Overall, $87.3 \%$ of the 17,520 high-quality sequences were longer than $300 \mathrm{bp}$. Cluster analyses assembled the 17,520 high-quality ESTs into 2,198 contigs and 11,709 singletons (13,907 unigenes, Table 1). The average length of the unigenes was $508 \mathrm{bp}$ (range from 100 to $1,367 \mathrm{bp}$ ).

\section{Unigene sequence analysis}

Unigenes were compared to annotations through the Gene Ontology Consortium using Interpro2GO. Graphs

Table 2 Partial unigenes with high homology to Homo sapiens known genes

\begin{tabular}{|c|c|c|}
\hline Query name & Annotation & Score \\
\hline gdtca_Cluster6467 & Homo sapiens cytoplasmic polyadenylation element binding protein 2 (CPEB2), transcript variant F, mRNA & 1037 \\
\hline gdtca_Cluster1457 & Homo sapiens zinc finger E-box binding homeobox 2 (ZEB2) on chromosome 2 & 944 \\
\hline gdtca_Cluster10676 & Homo sapiens TGF-beta activated kinase 1/MAP3K7 binding protein 3 (TAB3), mRNA & 914 \\
\hline gdtca_Cluster11351.seq.Contig1 & Homo sapiens splicing factor, arginine/serine-rich 12 (SFRS12), transcript variant 2, mRNA & 912 \\
\hline gdtca_Cluster8807 & Homo sapiens zinc finger protein 407 (ZNF407) on chromosome 18 & 892 \\
\hline gdtca_Cluster2284 & Homo sapiens SATB homebox 2 (SATB2) on chromosome 2 & 884 \\
\hline gdtca_Cluster4583 & Homo sapiens SAPS domain family, member 3 (SAPS3), transcript variant 3, mRNA & 846 \\
\hline gdtca_Cluster3803 & Homo sapiens LUC7-like 3 (S. cerevisiae) (LUC7L3), transcript variant 1, mRNA & 799 \\
\hline gdtca_Cluster4617 & Homo sapiens fibronectin type III and SPRY domain containing 1-like (FSD1L), transcript variant 3, mRNA & 797 \\
\hline gdtca_Cluster6088 & Homo sapiens nebulette (NEBL), transcript variant 3, mRNA & 783 \\
\hline gdtca_Cluster2858 & Homo sapiens formin-like 3 (FMNL3), transcript variant 2, mRNA & 773 \\
\hline gdtca_Cluster591 & Homo sapiens fat mass and obesity associated (FTO) on chromosome 16 & 765 \\
\hline gdtca_Cluster11523.seq.Contig1 & Homo sapiens Rho GTPase activating protein 19, mRNA (cDNA clone MGC:138804IMAGE:40082327) complete cds & 763 \\
\hline gdtca_Cluster11577.seq.Contig1 & Homo sapiens zinc finger CCCH-type containing 6 (ZC3H6), mRNA & 729 \\
\hline gdtca_Cluster8595 & Homo sapiens TEA domain family member 1 (SV40 transcriptional enhancer factor) (TEAD1), mRNA & 729 \\
\hline gdtca_Cluster6631 & Homo sapiens zinc finger and BTB domain containing 34 (ZBTB34), mRNA & 706 \\
\hline gdtca_Cluster1697 & Homo sapiens neuronal PAS domain protein 3 (NPAS3) on chromosome 14 & 686 \\
\hline gdtca_Cluster2466 & Homo sapiens SIX homeobox 1 (SIX1) on chromosome 14 & 682 \\
\hline gdtca_Cluster1883 & Homo sapiens B-cell CLL/lymphoma 11A (zinc finger protein) (BCL11A) on chromosome2 & 674 \\
\hline gdtca_Cluster8789 & Homo sapiens TAR DNA binding protein (TARDPB) on chromosome 1 & 650 \\
\hline gdtca_Cluster4024 & Homo sapiens protease, serine, 12 (neurotrupsin, motopsin) (PRSS12), mRNA & 636 \\
\hline gdtca_Cluster10567 & Homo sapiens fibroblast growth factor 14 (FGF14) on chromosome 13 & 620 \\
\hline gdtca_Cluster9160 & Homo sapiens forkhead box D3 (FOX3D) on chromosome 1 & 618 \\
\hline
\end{tabular}



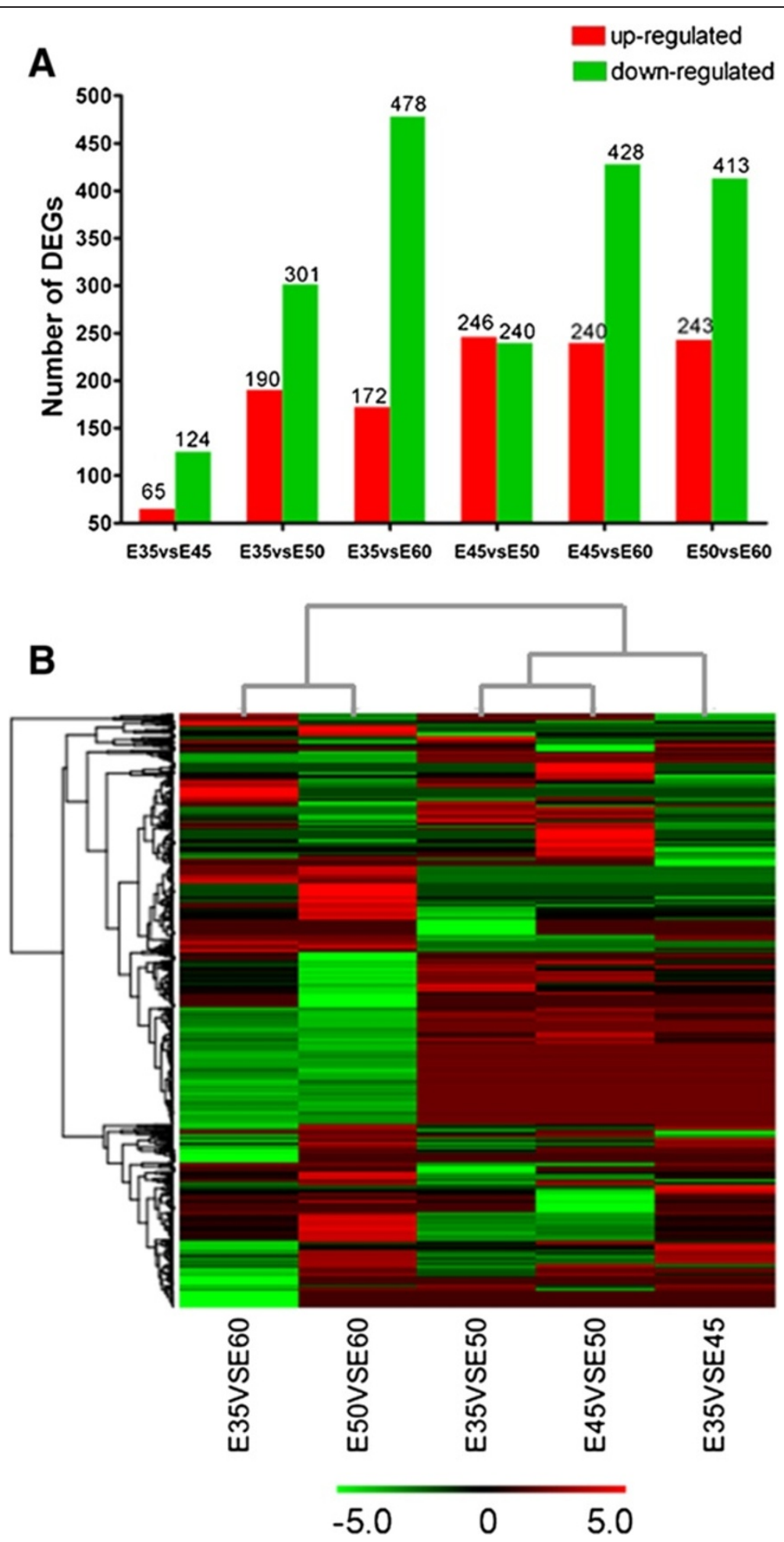

Figure 4 Genes from the four time periods were compared with each other. The differentially expressed genes from E35 vs E45, E35 vs E50, E35 vs E60, E45 vs E50, E45 vs E60, and E50 vs E60 were compared. (A) The number of up-regulated and down-regulated genes in each group is shown in the histogram. (B) The hierarchical clustering analysis of differentially expressed transcripts at different developmental stages.

based on the GO terms were created (Figure 3). Under the cellular component category, most transcripts were linked to inherent cellular structure, as well as to the protein complex (Figure 3A). In the category of molecular function, the five most abundant transcripts were involved in binding, catalytic activity, transporter activity, 
structural molecular activity, and signal transducer activity (Figure 3B). The most common biological processes were physiological processes and cellular processes (Figure 3C).

Based on BLAST results, $78.3 \%(10,883)$ of the unigenes were annotated to known genes, and $62.2 \%$ $(6,772)$ had a BLAST score greater than 200. There were 3,024 unknown unigenes $(21.7 \%)$ in the cDNA library. Unigenes whose sequences were markedly similar to known important proteins associated with dental development were found in this library, including ameloblastin, amelogenin, enamelin, dspp, and dmp1 (Additional file 6). What's more, expression of known specific transcription factors (Additional file 7), growth factors (Additional file 8), and related receptors (Additional file 9) during murine tooth development also can be searched in the cDNA library. These results indicated that the cDNA library will be useful in facilitating further dental experiments in the miniature pig model.

Homology searches showed that the top ten species were as follows: Sus scrofa (5,771), Homo sapiens $(2,122)$, Bos taurus (894), Equus caballus (467), Pan troglodytes (310), Canis familiaris (263), Macaca mulatta (262), Pongo abelii (53), Felis catus (39), and mouse (33). In the Unigene homology to Homo sapiens, 139 clones exhibited significant similarities to known genes (score greater than 500). Table 2 shows 23 unigenes with high homology to known Homo sapiens genes.

\section{CDNA expression patterns during tooth development}

We found that some cDNA sequences in the library had high homology to known Homo sapiens genes. Because detailed descriptive information concerning tooth development in Homo sapiens is lacking, miniature pigs are an optimal choice as a large animal model to investigate these molecular mechanisms. First, we compared all the four time periods with each other. The most differentially expressed genes were found between E35 and E60, followed by the genes between E45 and E60 (Figure 4A). And the fold change of all transcripts at E45 and E60 were the most significant (Figure 4B). Considering the germ tissue samples from E35 were small, and may have contained tissue from other nearby tissues, we blasted all the cDNA clones from the E45 and E60 time points against human genome DNA libraries. Twelve highly conserved genes were up-regulated (Table 3) and 15 highly conserved genes were down-regulated (Table 4). These results suggest that the 27 highly conserved genes may be involved in both miniature pig and Homo sapiens tooth development. Furthermore, 6 down-regulated (DPY30, ENAH, BORA, DAZAP2, NOP2, and DDX24) and 2 up-regulated genes (SHANK2, and CAMK2N1) in miniature pigs had higher homology to Homo sapiens genes compared with those in the mouse (Table 5).

\section{Discussion}

In the present study, we constructed a cDNA library from miniature pig molar tissue over the period of tooth development. We then confirmed the fold change of

Table 3 Up-regulated genes from E45 to E60 highly conserved homologous with known Homo sapiens genes

\begin{tabular}{|c|c|c|c|c|c|c|}
\hline Gene & TPM-E45 & TPM-E60 & log2 Ratio(E60/E45) & P-Value & FDR & Annotations \\
\hline gdtca_Cluster11155.seq.Contig1 & 8.33 & 51.11 & 2.61722 & 0 & 0 & $\begin{array}{l}\text { gi|119599067|schwannomin interacting } \\
\text { protein 1, isoform CRA_c [Homo sapiens] }\end{array}$ \\
\hline gdtca_Cluster8257 & 36.89 & 102.71 & 1.47727 & 0 & 0 & $\begin{array}{l}\text { gi|169161838|similar to hCG2040565 } \\
\text { [Homo sapiens] }\end{array}$ \\
\hline gdtca_Cluster3981 & 0.01 & 19.37 & 10.91961 & $2.22 \mathrm{E}-16$ & $2.96 \mathrm{E}-15$ & $\begin{array}{l}\text { gi|254911081|SH3 and multiple ankyrin } \\
\text { repeat domains } 2 \text { (SHANK2), transcript } \\
\text { variant 2, mRNAC1 [Homo sapiens] }\end{array}$ \\
\hline gdtca_Cluster13121.seq.Contig1 & 49.06 & 101.57 & 1.04986 & $3.18 \mathrm{E}-13$ & $3.26 \mathrm{E}-12$ & $\begin{array}{l}\text { gi|119604964|hypothetical protein MGC2747, } \\
\text { isoform CRA_c [Homo sapiens] }\end{array}$ \\
\hline gdtca_Cluster11734.seq.Contig1 & 30.68 & 91.48 & 1.57616 & $5.29 \mathrm{E}-13$ & $5.29 \mathrm{E}-12$ & $\begin{array}{l}\text { gi|119588946|hCG1992991, isoform CRA_a } \\
\text { [Homo sapiens] }\end{array}$ \\
\hline gdtca_Cluster11879.seq.Contig1 & 12.96 & 28.65 & 1.14447 & $1.12 \mathrm{E}-10$ & $9.21 \mathrm{E}-10$ & gi|119574191|hCG1983891 [Homo sapiens] \\
\hline gdtca_Cluster7989 & 0.01 & 4.39 & 8.77808 & $3.49 \mathrm{E}-10$ & 2.78E-09 & gi|10834656|PP2281 [Homo sapiens] \\
\hline gdtca_Cluster9754 & 3.97 & 13.35 & 1.74963 & 1.36E-09 & $1.01 \mathrm{E}-08$ & gi|7959776|PRO1489 [Homo sapiens] \\
\hline gdtca_Cluster11696.seq.Contig1 & 11.77 & 24.9 & 1.08103 & 9.32E-09 & 6.53E-08 & $\begin{array}{l}\text { gi|221046286|unnamed protein product } \\
\text { [Homo sapiens] }\end{array}$ \\
\hline gdtca_Cluster6950 & 2.64 & 13.84 & 2.39023 & $2.22 \mathrm{E}-08$ & $1.51 \mathrm{E}-07$ & $\begin{array}{l}\text { gi|226528280|short coiled-coil protein isoform } 1 \\
\text { [Homo sapiens] }\end{array}$ \\
\hline gdtca_Cluster5165 & 0.01 & 1.95 & 7.60733 & $5.90 \mathrm{E}-05$ & 0.00027 & gi|187957136|LOC730130 protein [Homo sapiens] \\
\hline gdtca_Cluster13166.seq.Contig1 & 1.85 & 5.7 & 1.62344 & 0.00018 & 0.0007 & gi|6653742|7h3 protein [Homo sapiens] \\
\hline
\end{tabular}


Table 4 Down-regulated genes from E45 to E60 highly conserved homologous with known Homo sapiens genes

\begin{tabular}{|c|c|c|c|c|c|c|}
\hline Gene & TPM-E45 & TPM-E60 & log2 Ratio(E60/E45) & P-Value & FDR & Annotations \\
\hline gdtca_Cluster2986 & 2338.42 & 250.01 & -3.22548 & 0 & 0 & $\begin{array}{l}\text { gi|37953286| transforming growth factor, } \\
\text { beta } 2 \text { (TGFB2) [Homo sapiens] }\end{array}$ \\
\hline gdtca_Cluster5775 & 6681.11 & 1182.18 & -2.49864 & 0 & 0 & $\begin{array}{l}\text { gi|62897645|eukaryotic translation elongation } \\
\text { factor } 1 \text { alpha } 1 \text { variant [Homo sapiens] }\end{array}$ \\
\hline gdtca_Cluster6915 & 1033.21 & 228.2 & -2.17876 & 0 & 0 & gi|119625564|hCG1820575 [Homo sapiens] \\
\hline gdtca_Cluster9994 & 176.92 & 24.09 & -2.87659 & $6.37 \mathrm{E}-190$ & $2.66 \mathrm{E}-188$ & $\begin{array}{l}\text { gi|168984469|retinoblastoma binding protein } 7 \\
\text { [Homo sapiens] }\end{array}$ \\
\hline gdtca_Cluster6869 & 141.88 & 22.95 & -2.62811 & 7.23E-139 & $2.68 \mathrm{E}-137$ & $\begin{array}{l}\text { gi| } 14211889 \mid \text { protein dpy-30 homolog } \\
\text { [Homo sapiens] }\end{array}$ \\
\hline gdtca_Cluster12512.seq.Contig1 & 23.4 & 0.65 & -5.16993 & $3.96 \mathrm{E}-40$ & $8.93 E-39$ & $\begin{array}{l}\text { gi| } 119627667 \mid \text { poly(A) binding protein, } \\
\text { cytoplasmic } 4 \text { (inducible form), isoform CRA_b } \\
\text { [Homo sapiens] }\end{array}$ \\
\hline gdtca_Cluster12827.seq.Contig1 & 41.12 & 10.42 & -1.98048 & $6.12 \mathrm{E}-30$ & $1.15 \mathrm{E}-28$ & $\begin{array}{l}\text { gi|4507797|ubiquitin-conjugating enzyme E2v2 } \\
\text { [Homo sapiens] }\end{array}$ \\
\hline gdtca_Cluster12105.seq.Contig1 & 22.21 & 3.42 & -2.69914 & $1.14 \mathrm{E}-23$ & $1.87 \mathrm{E}-22$ & $\begin{array}{l}\text { gi|242380880|hypothetical protein } \\
\text { [Homo sapiens] }\end{array}$ \\
\hline gdtca_Cluster12989.seq.Contig2 & 29.88 & 7.32 & -2.02926 & $8.32 \mathrm{E}-23$ & $1.34 \mathrm{E}-21$ & $\begin{array}{l}\text { gi|34533983|unnamed protein product } \\
\text { [Homo sapiens] }\end{array}$ \\
\hline gdtca_Cluster12482.seq.Contig1 & 6.35 & 0.01 & -9.31061 & 4.44E-13 & $4.48 \mathrm{E}-12$ & $\begin{array}{l}\text { gi|166014265|enabled-like protein variant } \\
\text { hMenaDv6 [Homo sapiens] }\end{array}$ \\
\hline gdtca_Cluster5336 & 16.26 & 4.72 & -1.78447 & $3.12 \mathrm{E}-11$ & $2.64 \mathrm{E}-10$ & $\begin{array}{l}\text { gi|158256424|unnamed protein product } \\
\text { [Homo sapiens] }\end{array}$ \\
\hline gdtca_Cluster5548 & 9.26 & 1.95 & -2.24754 & 7.36E-09 & 5.19E-08 & gi|4929627|CGI-79 protein [Homo sapiens] \\
\hline gdtca_Cluster11214.seq.Contig1 & 4.63 & 0.49 & -3.2401585391 & $9.20 \mathrm{E}-07$ & $5.23 \mathrm{E}-06$ & $\begin{array}{l}\text { gi|211904140|DAZ-associated protein } 2 \text { isoform c } \\
\text { [Homo sapiens] }\end{array}$ \\
\hline gdtca_Cluster8769 & 8.86 & 2.77 & -1.67742 & 2.96E-06 & $1.59 \mathrm{E}-05$ & $\begin{array}{l}\text { gi|119578911|nuclear transcription factor, X-box } \\
\text { binding 1, isoform CRA_a [Homo sapiens] }\end{array}$ \\
\hline gdtca_Cluster919 & 7.93 & 2.93 & -1.43642 & 7.92E-05 & 0.00035 & $\begin{array}{l}\text { gi|119609186|nucleolar protein 1, 120kDa } \\
\text { [Homo sapiens] }\end{array}$ \\
\hline
\end{tabular}

gene expression using qRT-PCR. Using large-scale sequencing and ESTs assemblage, a large pool of unigenes were found in this library. A total of 13,907 unigenes were assembled from 17,520 ESTs, indicating that redundancy was only $20.6 \%$. Furthermore, $95 \%$ of these Unigenes contain only one or two ESTs, indicating the positive effect of cDNA library normalization, which can be used to identify expressed genes in the future.

Table 5 Genes only expressed in Homo sapiens during the tooth development course of miniature pigs

\begin{tabular}{lll}
\hline & Query & Gene \\
\hline Down-regulation & gdtca_Cluster6869 & DPY30 \\
& gdtca_Cluster12482.seq.Contig1 & ENAH \\
& gdtca_Cluster5336 & BORA \\
& gdtca_Cluster11214.seq.Contig1 & DAZAP2 \\
& gdtca_Cluster919 & NOP2 \\
& gdtca_Cluster9198 & DDX24 \\
Up-regulation & gdtca_Cluster3981 & SHANK2 \\
& gdtca_Cluster9754 & CAMK2N1 \\
\hline
\end{tabular}

Great progress has been made in the study of molecular mechanisms during tooth morphogenesis in the past 20 years, and most data were derived from studies on rodent embryos [19]. However, owing to its similarity to human anatomy and physiology, pig models are superior in many aspects for the study of human development, diseases, and pre-clinical therapies [4-6]. Both domestic pigs and miniature pigs can be used in medical experimentation, but miniature pigs have many advantages, including an inherently small size, early sexual maturity, rapid breeding, and ease of management [25,26]. The deciduous molar in the Chinese experimental miniature pig is oblong in shape and has five or six main cusps. It is bigger and has different morphology compared with all other deciduous teeth in the mandible, and it lies on the end of mandible body. All these characteristics contribute to being able to easily and accurately distinguish and isolate the tooth germ. There are high correlations between the deciduous and permanent teeth [27]. Therefore, the deciduous molar was chosen as the first model tooth to evaluate in miniature pig tooth development. 
There is little information concerning tooth development in large animal models [23,24]. Some sequences in this cDNA library had high similarity with proteins associated with dental development such as ameloblastin, amelogenin, enamelin, dspp, and dmp1 [28-31]. Many genes involved in tooth development remain to be identified. For example, unigenes with high homology to known Homo sapiens genes in this library included FOXD3, $S A T B 2, Z E B 2$ (Zinc finger E-box-binding homeobox 2 gene), etc. FOXD3, a member of the forkhead family of transcriptional regulations, plays a role in maintaining the epiblast and its derivatives and in establishing pluripotent ESC lines [32]. SATB2 is a recently cloned member of the family of special AT-rich binding proteins. Satb $2^{-/-\mathrm{m}_{i c e}}$ exhibit both craniofacial abnormalities that resemble those observed in humans carrying a SATB2 translocation and defects in osteoblast differentiation and function [33]. ZEB2 has been involved in Mowat-Wilson syndrome (MWS), a multiple congenital anomaly syndrome characterized by a distinct facial phenotype. MWS is caused by heterozygous mutations or deletions in ZEB2 [34].

Data from this study will facilitate further dental experiments in the miniature pig model. In the present study, we found that 12 up-regulated and 15 downregulated genes may be involved in the miniature pig's tooth development. We also found 6 down-regulated (DPY30, ENAH, BORA, DAZAP2, NOP2, and DDX24) and 2 up-regulated genes (SHANK2 and CAMK2N1) in miniature pigs with higher homology to Homo sapiens genes compared with those in the mouse. SHANK2 is a member of the Shank family of synaptic proteins that function as molecular scaffolds in the postsynaptic density [35]. CAMK2N1 (calcium/calmodulin-dependent protein kinase II) expresses at high levels in osteogenic cells, and may be a good marker of osteogenic differentiation in mesenchymal stem cells [36]. There is very little known about these genes and their roles in tooth development. Investigating the functions of these genes in tooth development in a swine model and humans will be of great interest.

In summary, we evaluated the histological features of miniature pigs' deciduous molar development and identified five primary phases. A miniature pig embryo tooth cDNA library was constructed, which contains approximately $3.0 \times 10^{5} \mathrm{cfu}$ with 17,520 high quality EST sequences and 13,907 unigenes. The established cDNA library provides the basis for further tooth development studies using this animal model.

\section{Conclusion}

Our results not only identify the specific transcriptome and cDNA profile in developing mandibular deciduous molars of the miniature pig, but also provide useful information for investigating the molecular mechanism of tooth development in the miniature pig.

\section{Availability of supporting data}

The supporting data is available in the Genebank. The library accession numbers is LIBEST_028375. And the Library name is developing mandibular deciduous molars of the miniature pig cDNA library.

\section{Additional files}

\begin{abstract}
Additional file 1: Primers used for qRT-PCR.
Additional file 2: Excised mandibular deciduous molar germs from E35 and to E45 in miniature pig embryos. (A, B) The miniature pig mandible at E35. Red circles indicate the mandibular deciduous molar area. (C) One side of the mandible of a E45 miniature pig embryo. (D) Stripped medial mandible. (E) Mandibular stripped of excess tissue. (F) Isolated mandibular deciduous molar germs. Green arrow indicates mandible; red arrow indicates germ.

Additional file 3: Agarose gel electrophoresis of total RNA. Total RNA was extracted from the mandibular deciduous molar germs at each developmental stage (E35, E45, E50, E60). Total RNA examined by electrophoresis on $1.1 \%$ agarose gels showed two bright bands at 285 rRNA and 18S rRNA; the former was equal to or more abundant than the latter, indicating that little or no RNA degradation or contamination occurred during isolation.

Additional file 4: Agarose gel electrophoresis of double-stranded cDNA after PCR. One $\mu \mathrm{g}(1 \mu \mathrm{l})$ of poly(A) ${ }^{+}$RNA was used as RNA template in first-strand synthesis. A volume of $2 \mu \mathrm{l}$ of single-stranded cDNA served as a template for primer-extension-based, second-stand synthesis using 21 thermal cycles. Lane M: DL2000 plus marker (Transgen, $5 \mu \mathrm{l}$ ). Lane 1: $5 \mu \mathrm{l}$ sample of the double-stranded CDNA product showing a smear ranging from 0.1 to $3 \mathrm{~kb}$.
\end{abstract}

Additional file 5: Agarose gel electrophoresis of the PCR products from randomly selected cDNA inserts (30 plaques) from the unamplified cDNA library. The size of PCR products were between $1 \sim 3 \mathrm{~kb}$ for 30 samples. Lane M: DL2000 plus marker (Transgen).

Additional file 6: Known specific protein matrix expression in mice searched in the cDNA library during tooth development.

Additional file 7: Known specific transcription factor expression in mice searched in the cDNA library during tooth development.

Additional file 8: Known growth factor expression in mice searched in the CDNA library during tooth development.

Additional file 9: Known related receptor expression in mice searched in the cDNA library during tooth development.

\section{Competing interests}

The authors declare that they have no competing interests.

\section{Authors' contribution}

TS, TW, FW, and AL participated in initial discovery and design. TS, FW, and YX performed the histology experiments and carried out the gene expression assays. TS, TW and SW analyzed the data and wrote the manuscript. ZF, DL, XW, SC, CZ, and JH participated in the collection of the data and performed the statistical analysis. SW conceived of the study, participated in its design and coordination and got funding. All authors read and approved the final manuscript.

\section{Acknowledgments}

This work was supported by Ministry of Science and Technology of China, and was funded by the National Program on Key Basic Research Project of China No. 2007CB947304 and 2010CB944801. The authors declare that they have no competing interest.

\section{Author details}

${ }^{1}$ Molecular Laboratory for Gene Therapy and Tooth Regeneration, Beijing Key Laboratory of Tooth Regeneration and Function Reconstruction, Capital Medical University School of Stomatology, Tian Tan Xi Li No.4, Beijing 
100050, China. ${ }^{2}$ Department of Stomatology, Beijing Tongren Hospital Affiliated to Capital Medical University, Dong Jiao Min Xiang No.1, Beijing 100730, China. ${ }^{3}$ Department of Biochemistry and Molecular Biology, Capital Medical University School of Basic Medical Sciences, Beijing 100069, China.

Received: 20 August 2013 Accepted: 9 April 2014

Published: 21 April 2014

\section{References}

1. Larsen $M O$, Rolin B: Use of the Gottingen miniature as a model of diabetes, with special focus on type 1 diabetes research. ILAR J 2004, 45(3):303-313

2. Schultze-Mosgau S, Schliephake H, Radespiel-Troger M, Neukam FW: Osseointegration of endodontic end-osseous cones: zirconium oxide vs titanurm. Oral Surg Oral Med Oral Pathol Oral Radiol Endod 2000, 89(1):91-98.

3. Chen L, Shi Q, Scharf SM: Hemodynamic effects of periodic obstructive apneas in sedated pigs with congestive heart failure. J App/ Physio/ 2000, 88(3):1051-1060.

4. Dixon JA, Spinale FG: Large animal models of heart failure: a critical link in the translation of basic science to clinical practice. Circ Heart Fail 2009, 2(3):262-271.

5. Markert M, Koschany A, Lueth $\mathrm{T}$ : Tracking of the liver for navigation in open surgery. Int J Comput Assist Radiol Surg 2010, 5(3):229-235.

6. van der Spoel TI, Jansen of Lorkeers SJ, Agostoni P, van Belle E, Gyöngyösi M, Sluijter JP, Cramer MJ, Doevendans PA, Chamuleau SA: Human relevance of pre-clinical studies in stem cell therapy: systematic review and meta-analysis of large animal models of ischaemic heart disease. Cardiovasc Res 2011, 91(4):649-658.

7. Bermejo A, Gonzalez O, Gonzalez JM: The pig as an animal model for experimentation on the temporomandibular articular complex. Oral Surg Oral Med Oral Pathol 1993, 75(1):18-23.

8. Ruehe B, Niehues S, Heberer S, Nelson K: Miniature pigs as an animal model for implant research: bone regeneration in critical-size defects. Oral Surg Oral Med Oral Pathol Oral Radiol Endod 2009, 108(5):699-706.

9. Wang SL, Liu Y, Fang D, Shi S: The miniature pig: a useful large animal model for dental and orofacial research. Oral Dis 2007, 13(6):530-537.

10. Büchter A, Kleinheinz J, Wiesmann HP, Kersken J, Nienkemper M, Weyhrother $\mathrm{H}$, Joos U, Meyer U: Biological and biomechanical evaluation of bone remodelling and implant stability after using an osteotome technique. Clin Oral Implants Res 2005, 16(1):1-8

11. Nkenke E, Lehner B, Fenner M, Roman FS, Thams U, Neukam FW, RadespielTröger M: Immediate versus delayed loading of dental implants in the maxillae of miniatures: follow-up of implant stability and implant failures. Int J Oral Maxillofac Implants 2005, 20(1):39-47.

12. Sonoyama W, Liu Y, Fang D, Yamaza T, Seo BM, Zhang C, Liu H, Stan G, Wang CY, Shi S, Wang S: Mesenchymal stem cell-mediated functional tooth regeneration in Swine. PLoS One 2012, 1(1):79-92.

13. Gao RT, Yan X, Zheng CY, Goldsmith CM, Afione A, Hai B, Xu JJ, Zhou J, Zhang CM, Chiorini JA, Baum BJ, Wang SL: AAV2-mediated transfer of the human aquaporin-1 CDNA restores fluid secretion from irradiated miniature pig parotid glands. Gene Ther 2011, 18(1):38-42.

14. Xu JJ, Zheng ZM, Fang DJ, Gao RT, Liu Y, Fan ZP, Zhang CM, Shi ST, Wang SL: Mesenchymal stromal cell-based treatment of jaw osteoradionecrosis in swine. Cell Transplant 2012, 21(8):1679-1686.

15. Li YS, Xu JJ, Mao LS, Liu Y, Gao RT, Zheng ZM, Chen WJ, Le A, Shi ST, Wang SL: Allogeneic mesenchymal stem cell-based therapy for bisphosphonate-related osteonecrosis of the jaw in swine. Stem Cells Dev 2013, 22(14):2047-2056.

16. Wei F, Song T, Ding G, Xu J, Liu Y, Liu D, Fan Z, Zhang C, Shi S, Wang S: Functional tooth restoration by allogeneic mesenchymal stem cell-based bio-root regeneration in Swine. Stem Cells Dev 2013, 22(12):1752-1762.

17. Thesleff $I$, Sharpe P: Signalling networks regulating dental development. Mech Dev 1997, 67(2):111-123.

18. Thesleff I: Epithelial-mesenchymal signalling regulating tooth morphogenesis. J Cell Sci 2003, 116:1647-1648.

19. Tucker A, Sharpe P: The cutting-edge of mammalian development; how the embryo makes teeth. Nat Rev Genet 2004, 5(7):499-508.

20. Fleischmannova J, Matalova E, Tucker AS, Sharpe P: Mouse models of tooth abnormalities. Eur J Oral Sci 2008, 116(1):1-10.
21. Li A, Song TL, Wang F, Liu DY, Fan ZP, Cheng S, Zhang CM, He JQ, Wang SL: MicroRNAome and expression profile of developing tooth germ in miniature pigs. PLoS One 2012, 7(12):e52256.

22. Wang F, Xiao J, Cong W, Li A, Song T, Wei F, Xu J, Zhang C, Fan Z, Wang S: Morphology and chronology of diphyodont dentition in miniature pigs, Sus Scrofa. Oral Oral Dis 2014, 20(4):367-379.

23. Stembírek J, Buchtová M, Král T, Matalová E, Lozanoff S, Míšek I: Early morpho-genesis of heterodont dentition in miniature gigs. Eur J Oral Sci 2010, 118(6):547-558.

24. Bivin WS, McClure RC: Deciduous tooth chronology in the mandible of the domestic pig. J Dent Res 1976, 55(4):591-597.

25. England DC, Winters LM, Carpenter LE: The development of a breed of miniature swine; a preliminary report. Growth 1954, 18(4):207-214.

26. Weaver ME, Jump EB, McKean CF: The eruption pattern of permanent teeth in miniature swine. Arch Oral Biol 1969, 14(3):323-331.

27. Burdi AR, Superstine J: Developmental correlations of the deciduous and permanent teeth during the human fetal period. J Dent Res 1977, 56(12):1468

28. Hirst KL, Simmons D, Feng J, Aplin H, Dixon MJ, MacDougall M: Elucidation of the sequence and the genomic organization of the human dentin matrix acidic phosphoprotein 1 (DMP1) gene: exclusion of the locus from a causative role in the pathogenesis of dentinogenesis imperfecta type II. Genomics 1997, 42(1):38-45.

29. Krebsbach PH, Lee SK, Matsuki Y, Kozak CA, Yamada KM, Yamada Y: Full-length sequence, localization, and chromosomal mapping of ameloblastin: a novel tooth-specific gene. J Bio/ Chem 1996, 271(8):4431-4435.

30. MacDougall M, Simmons D, Luan X, Nydegger J, Feng J, Gu TT: Dentin phosphoprotein and dentin sialoprotein are cleavage products expressed from a single transcript coded by a gene on human chromosome 4: dentin phosphoprotein DNA sequence determination. J Biol Chem 1997, 272(2):835-842.

31. lijima M, Fan D, Bromley KM, Sun Z, Moradian-Oldak J: Tooth enamel proteins enamelin and amelogenin cooperate to regulate the growth morphology of octacalcium phosphate crystals. Cryst Growth Des 2010, 10(11):4815-4822.

32. Pohl BS, Knöchel W, Of Fox and Frogs: Fox (fork head/winged helix) transcription factors in Xenopus development. Gene 2005, 344:21-32.

33. Dobreva G, Chahrour M, Dautzenberg M, Chirivella L, Kanzler B, Fariñas I, Karsenty G, Grosschedl R: SATB2 is a multifunctional determinant of craniofacial patterning and osteoblast differentiation. Cell 2006, 125(5):971-986.

34. Garavelli L, Mainardi PC: Mowat-Wilson syndrome. Orphanet J Rare Dis 2007, 2:42.

35. Lim S, Naisbitt S, Yoon J, Hwang Jl, Suh PG, Sheng M, Kim E: Characterization of the Shank family of synaptic proteins. Multiple genes, alternative splicing, and differential expression in brain and development. J Biol Chem 1999, 74(41):29510-29518.

36. Ng F, Boucher S, Koh S, Sastry KS, Chase L, Lakshmipathy U, Choong C, Yang Z, Vemuri MC, Rao MS, Tanavde V: PDGF, TGF-beta, and FGF signaling is important for differentiation and growth of mesenchymal stem cells (MSCs): transcriptional profiling can identify markers and signaling pathways important in differentiation of MSCs into adipogenic, chondrogenic, and osteogenic lineages. Blood 2008, 112(2):295-307.

\section{doi:10.1186/1471-213X-14-16}

Cite this article as: Song et al:: Construction of a cDNA library for miniature pig mandibular deciduous molars. BMC Developmental Biology 2014 14:16 